\title{
Alterations in programmed cell death mechanism and their role in the pathogenesis of inflammatory bowel diseases
}

\author{
Piotr Eder, Liliana Łykowska-Szuber, Kamila Stawczyk-Eder, Iwona Krela-Kaźmierczak, Krzysztof Linke \\ Department of Gastroenterology, Human Nutrition, and Internal Diseases, Poznan University of Medical Sciences, Poznan, \\ Poland
}

Prz Gastroenterol 2014; 9 (5): 275-279

DOI: $10.5114 / p g .2014 .46162$

Key words: inflammatory bowel diseases, apoptosis, lymphocytes, enterocytes.

\begin{abstract}
Address for correspondence: Piotr Eder MD, PhD, Department of Gastroenterology, Human Nutrition, and Internal Diseases, Poznan University of Medical Sciences, 49 Przybyszewskiego St, 60-355, Poland, phone: +48 6186913 43, fax: +48 618691686 , e-mail: piotr.eder@op.pl
\end{abstract}

\begin{abstract}
Apoptosis plays an essential role in both physiology and pathology. In the pathogenesis of inflammatory bowel diseases, disturbances of apoptosis also play an important role. Inflammatory cells (for example lymphocytes, granulocytes) in the gut wall are resistant to apoptotic stimuli and they accumulate there causing tissue damage. On the other hand, apoptotic elimination of the enterocytes is enhanced, which leads to the impairment of the gut barrier. The exact mechanisms of these phenomena are still poorly understood and they are still under investigation. The present paper summarises current knowledge in terms of the role of alterations of programmed cell death in the pathogenesis of inflammatory bowel diseases.
\end{abstract}

\section{Introduction}

The aetiology of Leśniowski-Crohn's disease (CD) and ulcerative colitis (UC), both being inflammatory bowel diseases (IBD), is still unknown. Probably, the chronic inflammation in the intestinal wall in IBD results from an altered immune response to some poorly-defined antigens (probably of bacterial origin) in a genetically predisposed host exposed to several environmental factors [1, 2].

We still do not know which immunological defect initiates the chronic inflammatory cascade in IBD. There are some alterations in intestinal epithelial barrier functions described, in which different structural defects in tight junction proteins seem to play a crucial role. This leads to enhanced penetration of several antigens (toxins, microbes) to submucosal regions of the intestinal wall. There are also profound defects in the cooperation between subpopulations of T cells. The function of granulocytes, monocytes, and other cell populations of the innate immune system is also deeply disturbed. Among the most important disturbances from the pathological point of view are alterations in defensin production (also called endogenous antibiotics) in Paneth cells in the small intestinal epithelial layer. In recent years, a large number of studies have also shown that a crucial role in the pathogenesis of IBD is played by disturbances in cellular autophagy, in functions of the endoplasmic reticulum (ER) - the role of so-called ER stress. There are also data describing the pro-inflammatory influence of oxidative stress in the gut wall and the imbalance between several cytokines, as a key factors leading to perpetuation of chronic inflammation in IBD [3-5].

Most recent studies have shown that probably some bacterial antigens are responsible for the initiation of the inflammatory processes. It should be mentioned that the number of bacterial cells in the gut lumen is much higher than the number of all cells building the entire human body, so the intestinal immune system must be able to differentiate precisely pathogenic from physiological bacterial antigens [3, 4]. We hypothesise that disturbances in the immunological response to bacterial immune stimulation result in the pathological activation of several pro-inflammatory pathways. In the aforementioned mechanisms, the innate immune 
system cells are engaged as well as the cells belonging to the so-called adaptive immune response (mainly CD4 helper and CD8 cytotoxic lymphocytes) [5]. In CD, a predominance of Th1 - and Th17-dependent immune response is described, connected with enhanced activity of interleukin-2 (IL-2), IL-6, tumour necrosis factor- $\alpha$ (TNF- $\alpha$ ), interferon- $\gamma$ (IFN- $\gamma$ ), IL-17, IL-22, or IL-23. In UC, a predominance of Th2 response is observed - here the crucial role is played by IL-4, IL-5, or IL-10 $[6,7]$.

\section{Apoptosis - definition and mechanisms of action}

Disturbances in apoptosis (this process is also known as programmed cell death) seem to play an important role in IBD pathogenesis. This process in physiological conditions is strictly and precisely regulated, and it is energy dependent. Apoptotic elimination usually concerns single cells, and much more rarely some cellular groups. In contrast to necrosis, apoptosis does not generate an inflammatory reaction. Programmed cell death plays a crucial role both in physiological (for example in embryogenesis) and in many pathological conditions (primarily in oncogenesis and in inflammatory disorders) $[8,9]$.

Several pathways can finally lead to the initiation of cellular apoptosis. So-called extrinsic and intrinsic apoptosis induction pathways are the two best-characterised pathways. The extrinsic pathway is induced as a result of a stimulation of a death receptors' group. The best-studied death receptors are tumour necrosis factor receptor 1 (TNFR1), TNFR2, Fas (CD95), and death receptors 5 and 6 (DR5, DR6). The aforementioned receptors can be stimulated by ligands like TNF- $\alpha$ or Fas. The intrinsic pathway is induced by other factors, for example oxidative stress, ultraviolet radiation, some drugs, or disturbances in DNA structure and function. A crucial role in the intrinsic pathway is played by mitochondria. These organelles excrete to the cellular cytosol, promoting factors leading to the activation of this apoptotic pathway. Cytoplasmatic proteins belonging to the $\mathrm{Bcl}-2$ family play another important role in the apoptotic cascade. Some $\mathrm{Bcl}-2$ proteins enhance apoptosis (Bax, Noxa), while another subgroup has anti-apoptotic properties ( $\mathrm{Bcl}-2, \mathrm{Bcl}-\mathrm{W})$. A predominance of pro - or anti-apoptotic $\mathrm{Bcl}-2$ family proteins allows the initiation of programmed cell death or its blockade [10, 11].

In contrast to the different induction scenarios, the mechanisms of apoptotic execution phase are very similar in the majority of cell types. In this case, a crucial role is played by cysteine proteases called caspases. The members of this enzyme group undergo a cascade activation from inactive proenzymes to active particles. In the first phase, initiator caspases are activated (e.g. caspase 8,9 , or 10). At the final stage, so-called executive caspases (with crucial caspase 3 ) induce the final stages of cellular programmed cell death. The DNA is condensed (karyopyknosis) and then cut to similar fragments of roughly 180 base pairs, which are catalysed by specific endonucleases (karyorrhexis). The cell shrinks and the cellular membrane formulates so-called apoptotic bodies leading to cellular degradation. Apoptotic bodies contain DNA fragments and cellular organelles, the structure of which is not altered. Finally, apoptotic bodies are eliminated by phagocytic cells $[9,12]$.

\section{Disturbances of apoptosis in inflammatory bowel diseases}

As was mentioned above, disturbances in programmed cell death seem to play an important role in the immunopathogenesis of IBD. Apoptotic epithelial loss is enhanced, which leads to alterations of mucosal barrier functions. On the other hand, inflammatory cells are more resistant to apoptotic stimuli, which results in their accumulation in the intestinal wall [13].

In healthy intestinal tissues, inflammatory cascade, after being activated, undergoes self-limitation in two main pathways - anergy and apoptosis of immunoreactive cells (mainly T cells). In the first mechanism, cells are desensitised to multiple stimulatory ligands due to changes in the expression of membrane proteins engaged in intercellular interactions. As a result, the anergic cell becomes immunologically inactive. In the second mechanism, immune activated $T$ cells are eliminated due to interactions with other T-cell subpopulations. The extrinsic pathway related to the interaction between Fas protein on the target cell and FasL on the effector lymphocyte plays a crucial role $[14,15]$. It has been shown that lamina propria T cells (LPT) in healthy gut are more sensitive to pro-apoptotic stimuli than similar cells in the peripheral blood. This phenomenon is even multiplied after immunological stimulation [16]. It has been hypothesised that these mechanisms are related to a paradoxical influence of IL-2. This cytokine initially stimulates T-cell proliferation, but then it induces FasL expression, and in that way it contributes to cytotoxic reactions against reactive $T$ cells. In the last phase, the time-dependent silencing of IL-2 function, and lack of IL-2 stimulation, results in activation of apoptotic elimination of T cells [17].

These physiological mechanisms are altered in IBD, especially in CD. The first studies in this area came from Boirivant et al. They showed that LPTs from IBD patients were resistant to Fas-FasL-dependent apoptosis [18]. Additionally, it has been proven that LPT survival is prolonged in CD upon stimulation by CD2 (membrane protein of lymphocytes engaged in the intercellular 
interactions). Further research has shown that LPT apoptosis in UC is disturbed in a Fas-mediated manner, and probably the crucial phenomenon here is the high intracellular activity of FLIP protein, which inhibits an initiator, caspase 8 . This leads to disturbances in the first phases of apoptosis induction, and mitochondrial processes important for apoptosis regulation are not altered [19-21]. In CD, defects in programmed cell death of activated LPT seem to be more complicated and profound. Anti-apoptotic $\mathrm{BCl}-2$ proteins (e.g. $\mathrm{BCl}-2$, $\mathrm{Bcl}-\mathrm{x})$, which are expressed in lymphocytes in $\mathrm{CD}$ in high amounts, probably play a crucial role in these phenomena [22-24].

We still do not know if alterations of apoptotic lymphocyte elimination is a primary phenomenon or whether it is secondary to inflammatory lesions in the intestines in IBD. Research performed so far has suggested that apoptotic defects are present, probably due to high expression of pro-inflammatory cytokines like IL-6, IL-12, or TNF- $\alpha$, and/or due to disturbances in immunosuppressive mechanisms.

Interleukin-6 stimulates signal transducer and activator of transcription 3 (STAT3) pathway, which enhances the expression of anti-apoptotic $\mathrm{Bcl}-2$ family proteins [25-27]. These phenomena are observed in CD.

Interleukin-12 stimulates intercellular STAT-4 pathway, which inhibits the first phases of apoptotic extrinsic pathway [28]. These processes are observed first of all in UC patients.

Tumour necrosis factor- $\alpha$ is also present at inflammatory sites in high amounts. TNFR1 and TNFR2 are specific receptors for this cytokine. TNFR1 is present on almost all cell types and interacts with the highest affinity with the soluble form of TNF- $\alpha$ - STNF [29]. Stimulation of TNFR1 can have pleiotropic effects. The final phenomenon induced by TNF-TNFR1 interaction is dependent mainly on the presence of other cofactors (e.g. other cytokines), and on the metabolic activity of the target cell. TNFR2 is mainly expressed on haematopoietic and endothelial cells, and it interacts with the transmembrane TNF- $\alpha-$ tmTNF. TNFR2 can support TNFR1 functions in the so-called ligand-passing mechanism [29]. TNFR2 can also participate in the stimulation of CD4 cells by macrophages to excrete an anti-apoptotic IL-6 [30]. Both receptors, TNFR1 and TNFR2, also have soluble forms in intercellular space. They probably inactivate TNF- $\alpha$, and thus they cooperate in the modulation of immune processes [14].

In chronic inflammatory conditions, e.g. in IBD, interaction between TNF- $\alpha$ and TNFR1 can lead to stimula-

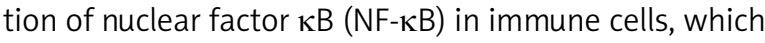
promotes transcription of genes, leading to cellular proliferation. Nuclear factor $\kappa \mathrm{B}$ pathway can also promote predominance of anti-apoptotic $\mathrm{Bcl}-2$ family proteins [31]. Another possible link between NF- $\mathrm{B} B$ activity and promotion of inflammatory lesions in IBD is the alteration in function of immunosuppressive cells and cytokines. One of the most important immunosuppressive cellular populations whose function is disturbed is that of T regulatory (Treg) cells [24]. Treg contribute under physiological conditions to the inhibition of pro-inflammatory processes. They play a crucial role in immune tolerance against bacterial antigens of physiological intestinal microbial flora. The tolerance mechanisms are related to direct intercellular interactions but can also be mediated by immunosuppressive cytokines, e.g. IL-10 or transforming growth factor- $\beta$ (TGF- $\beta$ ). It has been shown, under experimental conditions, that stimulation of mononuclear cells by TGF- $\beta$ can lead to down-regulation of NF- $\kappa B$ stimulation via TNF- $\alpha$ [32]. There are also some alterations described in Treg functions in IBD. It has been shown that target pro-inflammatory lymphocytes are less sensitive to Treg influence. A high amount of TNF- $\alpha$ at the inflammatory site plays a crucial role in the aforementioned altered immunosuppressive mechanisms. The main factors, which are directly engaged in these phenomena, are TGF- $\beta$ inhibitors, e.g. Smad7 protein. The final result of these processes is the imbalance between pro-inflammatory and immunosuppressive factors, which promotes chronic inflammation in IBD.

Studies performed by Neurath et al. from Medical University in Erlangen, Germany provide very interesting results in terms of the mechanisms of apoptotic disturbances in IBD. They showed that probably the presence of monocytes in inflammatory infiltrates is crucial for the resistance to apoptosis of CD4 lymphocytes. Monocytes/macrophages, having a high expression of tmTNF, stimulate TNFR2 on CD4 lymphocytes. This results in activation of TRAF2 pathway and NF- $\kappa B$. These factors promote the synthesis of IL- 6 by CD4 cells, which in an autocrine-like manner induces resistance to pro-apoptotic factors in CD4 cells. The authors speculate that probably induction of apoptotic elimination of CD4 lymphocytes is crucial for the efficacy of anti-TNF antibodies, which are used in IBD therapy. The programmed cell death of pro-inflammatory cells is induced in this model by binding anti-TNF antibody to tmTNF on macrophages, which blocks the interaction between tmTNF with TNFR2 on CD4 cells [30].

In summary, the resistance to apoptosis of LPT together with a very high proliferative index of LPT in IBD (which is higher than in many other inflammatory diseases) results in accumulation of immunoreactive cells in intestinal tissues, which leads to tissue destruction and alters the function of the gut [20]. 
However, although disturbances in apoptosis in IBD are mainly observed in LPT, also other cellular populations are defective in terms of the regulation of the programmed cell death mechanism. Concerning intestinal epithelial cells, is has been shown that in IBD, their apoptotic elimination is enhanced, which leads to the impairment of intestinal barrier function. In physiological conditions, enterocytes belong to one of the most dynamically proliferating cells in the human body. Within the time of 3-5 days, a total restitution of the intestinal epithelial layer takes place. The epithelial germinal cells, which are present in the basal parts of the intestinal villi, play a crucial role in this phenomenon. They differentiate and migrate to the superficial parts of the villi, performing many important metabolic and immunological functions. After having their role fulfilled, they die in order to enable younger cells to perform their functions. Apoptosis seems to be crucial for these phenomena. It has been proven that epithelial programmed cell death takes place, first of all, in the apical parts of villi in the small intestine and in the superficial areas of the colonic epithelium. The direct mechanisms leading to these processes are not well known. It has been hypothesised that probably the loss of contact between the epithelial cells and the extracellular space proteins of the basement membrane activates the caspase pathways [13].

The pro-apoptotic ratio of epithelial cells is increased both in CD and UC, in comparison with the healthy gut. As has been mentioned above, this leads to impairment of the intestinal barrier function, which enables the penetration of several antigens (toxins, food antigens, bacterial antigens, etc.) to the submucosal areas of the gut wall. However, it should be mentioned that the programmed cell death of enterocytes seems to be more disturbed in UC. The majority of studies show that Fas-FasL interactions play a crucial role here. Fas is present on the epithelial cells (target cells), and LPTs in inflamed tissues present high amounts of FasL. In that way LPTs induce programmed cell death of enterocytes. The direct interaction in the Fas-FasL pathway between epithelial cells $[33,34]$ is another possible mechanism. However, it should be pointed out that recently the importance of the Fas-FasL pathway has been questioned by some authors. It has been suggested that the methodology of some studies on the role of the Fas-FasL mechanism in the induction of enterocyte apoptosis is controversial (e.g. the quality of part of the FasL-detecting antibody kits was questioned, and their withdrawal from the market was postulated) [35]. Thus, there are still some questions to be answered in this subject.

As far as CD is concerned, the most important studies on the apoptotic disturbances in epithelial cells come from Di Sabatino et al. In 2003 they neglected the role of the Fas-FasL mechanism in the enhancement of apoptotic elimination of epithelial cells [36]. The role of other pathways is still being investigated. One of the hypotheses is that disturbances in the interaction between enterocytes and the mucosal basement membrane proteins are the leading forces of those phenomena. Other hypotheses discuss the potential role of TNFRs. However, more data are needed in terms of the importance and the mechanisms of the apoptotic elimination of epithelial cells in CD [37-39].

In conclusion, it has to be emphasised that although our knowledge in terms of the pathogenesis of IBD is increasing, there are still more questions than answers in this area. The same concerns the role of apoptotic disturbances in IBD. It has been shown that these processes play a role in chronic inflammatory conditions like CD and UC. However, many of the studies on this subject were conducted on animal models or in cellular lines in vitro, so further studies on patients with IBD are still needed in order to have a closer look into the mechanisms of action of apoptotic cellular elimination, both in CD and UC.

\section{References}

1. Neumann MG. Immune dysfunction in inflammatory bowel disease. Translational Research 2007; 149: 173-86.

2. Cho JH, Brant SR. Recent insights into the genetics of inflammatory bowel disease. Gastroenterology 2011; 140: 1704-12.

3. Chassaing B, Darfeuille-Michaud A. The commensal microbiota and enteropathogens in the pathogenesis of inflammatory bowel diseases. Gastroenterology 2011; 140: 1720-8.

4. Abraham C, Medzhitov R. Interactions between the host innate immune system and microbes in inflammatory bowel disease. Gastroenterology 2011; 140: 1729-37.

5. Strober W, Fuss IJ. Proinflammatory cytokines in the pathogenesis of inflammatory bowel disease. Gastroenterology 2011; 140: 1756-67.

6. Sakuraba A, Sato T, Kamada N, et al. Th1/Th17 immune response is induced by mesenteric lymph node dendritic cells in Crohn's disease. Gastroenterology 2009; 137: 1736-45.

7. Stober W, Fuss IJ, Mannon P. The fundamental basis of inflammatory bowel disease. J Clin Invest 2007; 117: 514-21.

8. Danial NN, Korsmeyer SJ. Cell death: critical control points. Cell 2004; 116: 205-19.

9. Wójcik C. Apoptosis. In: Seminars in cytophysiology: textbook for medicine, veterinary, and biology students [Polish]. Kawiak J, Zabel M (eds.). Urban \& Partner, Wroclaw 2002; 88-102.

10. Ashkenazi A, Dixit VM. Apoptosis control by death and decoy receptors. Curr Opin Cell Biol 1999; 11: 255-60.

11. Lindsay J, Esposti MD, Gilmore AP. Bcl-2 proteins and mitochondria - specificity in membrane targeting for death. Biochim Biophys Acta 2011; 1813: 532-9.

12. Fan TJ, Han LH, Cong RS, et al. Caspase family proteases and apoptosis. Biochim Biophys Acta 2005; 37: 719-27. 
13. Edelblum KL, Yan F, Yamaoka T, et al. Regulation of apoptosis during homeostasis and disease in the intestinal epithelium. Inflamm Bowel Dis 2006; 12: 413-24.

14. Malejczyk J. Cytotoxic lymphocytes - mechanisms of action. In: Immunology [Polish]. Gołąb J, Jakóbisiak M, Lasek W (eds.). PWN, Warsaw 2004; 277-87.

15. MacDonald TT, Monteleone G. Immunity, inflammation, and allergy in the gut. Science 2005; 307: 1920-5.

16. Neurath MF, Finotto S, Fuss IJ, et al. Regulation of T-cell apoptosis in inflammatory bowel disease: to die or not to die, that is the mucosal question. Trends Immunol 2001; 22: 21-6.

17. Boirivant M, Pica R, De Maria R, et al. Stimulated human lamina propria $T$ cells manifest enhanced Fas-mediated apoptosis. J Clin Invest 1996; 98: 2616-22.

18. Boirivant M, Marini M, Di Felice G, et al. Lamina propria T cells in Crohn's disease and other gastrointestinal inflammation show defective CD2 pathway-induced apoptosis. Gastroenterology 1999; 116: 557-65.

19. Peppelenbosch MP, van Deventer SJH. T cell apoptosis and inflammatory bowel disease. Gut 2004; 53: 1556-8.

20. Sturm A, Leite AZ, Danese S, et al. Divergent cell cycle kinetics underlie the distinct functional capacity of mucosal T cells in Crohn's disease and ulcerative colitis. Gut 2004; 53: 1624-31.

21. Seidelin JB, Nielsen $\mathrm{OH}$. Attenuated apoptosis response to Fas-ligand in active ulcerative colitis. Inflamm Bowel Dis 2008; 14: $1623-9$

22. Itoh J, de la Motte C, Strong SA, et al. Decreased Bax expression by mucosal $T$ cells favours resistance to apoptosis in Crohn's disease. Gut 2001; 49: 35-41.

23. Ina K, Itoh J, Fukushima K, et al. Resistance of Crohn's disease T cells to multiple apoptotic signals is associated with $\mathrm{Bcl}-2 /$ Bax mucosal imbalance. J Immunol 1999; 163: 1081-90.

24. MacDonald TT, Monteleone I, Fantini MC, et al. Regulation of homeostasis and inflammation in the intestine. Gastroenterology 2011; 140: 1768-75.

25. Yamamoto M, Yoshizaki K, Kishimoto T, et al. IL-6 is required for the development of Th1 cell mediated murine colitis. J Immunol 2000; 164: 4878-82.

26. Jones SA, Richards PJ, Scheller J, et al. IL-6 transsignaling: the in vivo consequences. J Interferon Cytokine Res 2005; 25: 241-53.

27. Ito H. IL-6 and Crohn's disease. Curr Drug Targets Inflamm Allergy 2003; 2: 125-30.

28. Mudter J, Weigmann B, Bartsch B, et al. Activation pattern of signal transducers and activators of transcription (STAT) factors in inflammatory bowel diseases. Am J Gastroenterol 2005; 100: 64-72.

29. Eder P, Łykowska-Szuber L, Stawczyk-Eder K, et al. Blockers of tumour necrosis factor-alpha: mechanisms of action [Polish]. Prz Gastroenterol 2011; 5: 290-8.

30. Atreya R, Zimmer M, Bartsch B, et al. Antibodies against tumor necrosis factor (TNF) induce T-cell apoptosis in patients with inflammatory bowel diseases via TNF Receptor 2 and intestinal CD14+ macrophages. Gastroenterology 2011; 141: 2026-38.

31. Edelblum KL, Goettel JA, Koyama T, et al. TNFR1 promotes tumor necrosis factor-mediated mouse colon epithelial cel survival through RAF activation of NF-kappaB. J Biol Biochem 2008; 283: 29485-94.
32. Monteleone G, Mann J, Monteleone I, et al. A failure of transforming growth factor-beta1 negative regulation maintains sustained NF-kappaB activation in gut inflammation. J Biol Chem 2004; 279: 3925-32.

33. Iwamoto M, Koji T, Makiyama K, et al. Apoptosis of crypt epithelial cells in ulcerative colitis. J Pathol 1996; 180: 152-9.

34. Ueyama H, Kiyohara T, Sawada N, et al. High Fas ligand expression on lymphocytes in lesions of ulcerative colitis. Gut 1998; 43: 48-55.

35. Chen L, Park SM, Turner JR, et al. Cell death in the colonic epithelium during inflammatory bowel diseases: CD95/Fas and beyond. Inflamm Bowel Dis 2010; 16: 1071-6.

36. Di Sabatino A, Ciccocioppo R, Luinetti O, et al. Increased enterocyte apoptosis in inflamed areas of Crohn's disease. Dis Colon Rectum 2003; 46: 1498-507.

37. Von Lampe B, Barthel B, Coupland SE, et al. Differential expression of matrix metalloproteinases and their tissue inhibitors in colon mucosa of patients with inflammatory bowel disease. Gut 2000; 47: 63-73.

38. Foster DM, Stauffer SH, Stone MR, et al. Proteasome inhibition of pathologic shedding of enterocytes to defend barrier function requires $X$-linked inhibition of apoptosis and nuclear factor kappaB. Gastroenterology 2012; 143: 133-44.

39. Siggers RH, Hackam DJ. The role of innate immune-stimulated epithelial apoptosis during gastrointestinal inflammatory diseases. Cell Mol Life Sci 2011; 68: 3623-34.

Received: 4.05 .2012

Accepted: 25.11 .2012 